\title{
Effect of Spacing on Flow Field Characteristic of Tube Artificial Reefs with Parallel Formation by CFD
}

\author{
${ }^{1}$ Mohammad Tauviqirrahman, ${ }^{1}$ Sugiyanto, ${ }^{1}$ Eflita Yohana, ${ }^{1}$ Mulyadi, ${ }^{1}$ Jamari and ${ }^{2}$ Muchammad \\ ${ }^{1}$ Department of Mechanical Engineering, Faculty of Engineering, Diponegoro University, \\ Semarang, Central Java, Indonesia \\ ${ }^{2}$ Laboratory for Surface Technology and Tribology, Faculty of Engineering Technology, \\ University of Twente, Drienerloolan 5, Postbus 217, 7500 AE Enschede, Netherlands
}

\begin{abstract}
In addition to attracting to fish, the flow field characteristics around Artificial Reefs (ARs) are key factors to prevent beach erosion. In the present study, research on hydrodynamics of artificial reefs primarily focusing on numerical simulation is conducted. The tube artificial reefs with parallel formation is analyzed based on comprehensive three-dimensional Computational Fluid Dynamic (CFD) approach by Renormalization Group (RNG) $k-\varepsilon$ turbulent model with respect to the length of slow flow region. The arrangement of artificial reefs is applied varying the spacing between the AR units both in transversal and longitudinal direction. The numerical results indicate that a greater artificial reef effect dealing with the slow flow region is obtained when the distance between the AR units of artificial reef is relatively close. According to the results, the largest slow flow region is achieved when the three rows of AR units is used. This result can be considered as a guide to reduce the beach erosion.
\end{abstract}

Key words: Abrasion, Artificial Reef (AR), Computational Fluid Dynamic (CFD), slow flow region, transversal, longitudinal

\section{INTRODUCTION}

Artificial Reefs (ARs) have been extensively used to increase total biomass production including fish when they are deployed in the sea (Bohnsack and Sutherland, 1985). Recent studies have presented the significant effect of flow fields around reefs from the perspective of ecological term and hydrodynamic term. Based on hydrodynamic point of view of the artificial reef, Ontowirjo and Armono (2003) using a numerical modeling approach observed the hydrodynamic parameters (i.e., particle velocities, fluid flows, wave breaking and dissipation of wave energy) of a, specially, shaped submerged structure to reduce the offshore waves energy as well as to provide a safe and productive environment for fish. Miao and Xie (2007) studied the effects of water-depth on the hydrodynamic force of the artificial reef by simulating regular and irregular waves. They found that in the ultra-shallow water hydrodynamic force increases very evidently with the decrease of water-depth. Jiang et al. $(2010,2013)$ developed a numerical method to investigate the hydrodynamic characteristic of artificial reef and found that the numerical simulation results are in a good agreement with results observed by experimental means. Thus, they suggested that the numerical simulation method can be applied to predict the hydrodynamic behavior associated with artificial reefs. Other interesting result was drawn by Woo et al. (2014). They proposed a numerical method which can be applied to predict the drag coeffcients of the artificial reefs. Later, more works have been conducted by Kim et al. (2014) focusing not only for drag coefficients but also for wake region and structural response of the general ARs to make more attractions to marine bio creatures and the stability in water flow.

The issue of what shape of artificial reef can affect very much the flow fields has also attracted significant attention from engineers and marine ecologists. Liu et al. (2012) used the numerical simulation and experiment methods to study the flow fields within and around a hollow cube artificial reef varying the sizes of holllows. Later, Liu et al. (2013) studied the flow field characteristics around star-shaped artificial reefs. In their research, they discussed the influence of arrangement and spacing on

Corresponding Author: Mohammad Tauviqirrahman, Department of Mechanical Engineering, Faculty of Engineering, Diponegoro University, Semarang, Central Java, Indonesia 
the flow field of one and two artificial reefs. In recent publication, Jiao et al. (2017) studied the effect of flow fields around tube artificial reefs based on the numerical simulation and PIV experimental. It was found that a greater unit artificial reef effect is obtained when five reefs are involved and the ratio of reef unit height to water depth is 0.224 . In addition, some researchers also showed that the flow field could be affected by the arrangement of the artificial reefs (Suzuki et al., 2011; Liu and Su, 2013).

Based on literature survey mentioned, it seems that most of studies focused on the deployment of the artificial reef for coral restoration. Therefore, in the current study based on the hydrodynamic point of view, it is essential issue to investigate the flow field within and around the artificial reefs with respect to the enhancement of the artificial reefs performance for preventing beach abrasion. The main idea of this research is how the Artificial Reef (AR) arrangement could generate the longest slow flow region.

Following the main frame of this idea with respect to the flow characteristic around the Artificial Reef (AR), the investigation of the effect of ARs arrangement on the flow field and how spacing affects the flow field in detail is carried out. For computation, the ARs are characterized by various rows of tube ARs varying their spacing both in longitudinal or transversal direction. The length of the slow flow region is of particular interest. The numerical model is based on a solution of the equations governing momentum and conservation of mass for a incompressible, Newtonian fluid based on Computational Fluid Dynamic (CFD).

\section{MATERIALS AND METHODS}

Governing equations: The water flow in the simulation is assumed to consist of an incompressible and viscous Newtonian fluid with a constant density. The flow is in an unsteady state and without heat exchanges. The velocity calculations of water flow are based on the continuity equation. The three-dimensional Reynolds-Average Navier-Stokes (RANS) equation is employed as the momentum equation. The equations are listed as follows:

The momentum equation:

$$
\frac{\partial}{\partial \mathrm{t}} \rho\left(\rho \mathrm{u}_{\mathrm{i}}\right)+\frac{\partial}{\partial \mathrm{x}_{\mathrm{i}}}\left(\rho \mathrm{u}_{\mathrm{i}} \mathrm{u}_{\mathrm{j}}\right)=-\frac{\partial \mathrm{p}}{\partial \mathrm{x}_{\mathrm{i}}}+\frac{\partial}{\partial \mathrm{x}_{\mathrm{j}}}\left[\mu \frac{\partial \mathrm{u}_{\mathrm{i}}}{\partial}-\overline{\overline{\mathrm{u}_{\mathrm{i}}^{\prime} \mathrm{u}_{\mathrm{j}}^{\prime}}}\right]+\mathrm{S}_{\mathrm{i}}(1)
$$

The continuity equation:

$$
\frac{\partial \rho}{\partial t}+\frac{\partial}{\partial x_{i}}\left(\rho u_{i}\right)=0
$$

In Eq. 1 and 2 is the density of the fluid; $u_{i}$ and $\mathrm{u}_{\mathrm{j}}$ are the average velocity components for $\mathrm{x}, \mathrm{y}, \mathrm{z} ; \rho$ is the static hydrodynamic pressure; $\mu$ is the viscosity; $\mathrm{u}_{i}^{\prime}$ and $u_{j}^{\prime}$ are the fluctuation velocities; $-\rho \overline{u_{i}^{\prime} u_{j}^{\prime}}$ is the Reynolds stress; $i, j=1,2,3(x, y, z)$ and $S_{i}$ is the source item.

In the present study, the Boussinesq hypothesis is assumed to establish the relationships between Reynolds stresses and mean velocity gradients. The equation of Reynolds stresses is given by Eq. 3 :

$$
\overline{-\rho u_{i}^{\prime} u_{j}^{\prime}}=\mu_{t}\left(\frac{\partial u_{i}}{\partial x_{j}}+\frac{\partial u_{j}}{\partial x_{i}}\right)-\frac{2}{3}\left(\rho k+\mu_{t} \frac{\partial u_{i}}{\partial x_{i}}\right) d_{i j}
$$

Where:

$\mu_{\mathrm{t}}=$ The turbulent viscosity

$\mathrm{k}=$ The turbulent kinetic energy

Turbulence model: In the present research, the Renormalisation Group (RNG) $k-\varepsilon$ turbulence model is adopted. This is an improved version of the $k-\varepsilon$ turbulence model. The new turbulent equations for " $\mathrm{k}$ " and " $\varepsilon$ " are listed as follows:

k equation:

$$
\frac{\partial(\rho \mathrm{k})}{\partial \mathrm{t}}+\frac{\partial\left(\rho \mathrm{ku}_{\mathrm{i}}\right)}{\partial \mathrm{x}_{\mathrm{i}}}=\frac{\partial}{\partial \mathrm{x}_{\mathrm{j}}}\left[\mathrm{a}_{\mathrm{k}} \mu_{\mathrm{eff}} \frac{\partial \mathrm{k}}{\partial \mathrm{x}_{\mathrm{j}}}\right]+\mathrm{G}_{\mathrm{k}}+\rho \mathrm{e}
$$

$\varepsilon$ equation:

$$
\frac{\partial(\rho e)}{\partial t}+\frac{\partial\left(\rho_{e u}\right)}{\partial x_{i}}=\frac{\partial}{\partial x_{j}}\left[a_{k} \mu_{e f f} \frac{\partial e}{\partial x_{j}}\right]+C_{1 e}^{*} \frac{e}{k} G_{k}-C_{2 e}^{*} \rho \frac{e^{2}}{k}
$$

In Eq. 4 and $5, G_{\mathrm{k}}$ is expressed by the average flow gradient based on the turbulent kinetic energy, $\mu_{\text {eff }}$ is the validity turbulent viscosity coefficient, $\alpha_{k}$ and $\alpha_{\varepsilon}$ inverse turbulent Prandtl numbers for the $\mathrm{k}$ and $\varepsilon$ equations and $\mathrm{C}_{1 \mathrm{c}}$ and $\mathrm{C}_{2 \mathrm{c}}$ are known constants. The calculated equations of these items and coefficients are expressed as:

$$
\begin{aligned}
& \mathrm{G}_{\mathrm{k}}=\mu_{\mathrm{t}}\left(\frac{\partial \mathrm{u}_{\mathrm{i}}}{\partial \mathrm{x}_{\mathrm{j}}}+\frac{\partial \mathrm{u}_{\mathrm{j}}}{\partial \mathrm{x}_{\mathrm{i}}}\right) \frac{\partial \mathrm{u}_{\mathrm{i}}}{\partial \mathrm{x}_{\mathrm{j}}}, \mu_{\text {eff }}=\mu+\mu_{\mathrm{t}}, \\
& \mu_{\mathrm{t}}=\rho \mathrm{C}_{\mu} \frac{\mathrm{k}^{2}}{\mathrm{e}}, \mathrm{C}_{\mu}=0.0845
\end{aligned}
$$




$$
\begin{aligned}
& \mathrm{a}_{\mathrm{k}}=\mathrm{a}_{\mathrm{e}}=1.390, \mathrm{C}_{1 \mathrm{e}}^{*}=\mathrm{C}_{1 \mathrm{e}}- \\
& \frac{\eta\left(1-\eta / \eta_{0}\right)}{1+\beta \eta^{3}}, \mathrm{C}_{1 \mathrm{e}}=1.42, \mathrm{C}_{2 \mathrm{e}}=1.68 \\
& \eta_{0}=4.377, \beta=0.012, \eta= \\
& \left(2 \mathrm{E}_{\mathrm{ij}} \times \mathrm{E}_{\mathrm{ij}}\right)^{1 / 2} \frac{\mathrm{k}}{\mathrm{e}}, \mathrm{E}_{\mathrm{ij}}=\frac{1}{2}\left(\frac{\partial \mathrm{u}_{\mathrm{i}}}{\partial \mathrm{x}_{\mathrm{j}}}+\frac{\partial \mathrm{u}_{\mathrm{j}}}{\partial \mathrm{x}_{\mathrm{i}}}\right)
\end{aligned}
$$

Where:

$$
\begin{aligned}
& \eta=\text { A dimensionless parameter } \\
& \eta_{0}, C_{\mu}=\text { Constants } \\
& \text { and } \beta \\
& E_{i j}=\text { The main rate of the strain tensor }
\end{aligned}
$$

CFD Model and boundary conditions: In this study, the computational domain representing the beach area containing the schematic of Artificial Reefs (AR) is shown in Fig. 1. Whilst, the parameters used are defined in detail in Table 1. It should be noted that all of the variables in Table 1 is defined in $\mathrm{D}$ in which $\mathrm{D}$ is the diameter of artificial reef unit. The domain consists of three rows of artificial reef parallelly deployed at certain distance from the beach line. In the present study, the number of AR row is varied (in this case 1-3 rows). For all following computations in each row $5 \mathrm{AR}$ units are implemented. It should be noted that the value of the width of the domain $\left(\mathrm{L}_{\mathrm{p}}\right)$ depends on the spacing between $\mathrm{AR}$ units in transversal direction $\left(\mathrm{A}_{c}\right)$ as shown in Fig. 2. In the present study, the $R_{c}$ and $A_{c}$ as shown in Fig. 2 are varied with respect to the velocity distribution surrounding reefs. In this way, the optimal formation of ARs can be obtained. This variation is performed to obtain the most effective formation of AR dealing with the slow flow region. The CFD analysis is performed using ANSYS Fluent 7. The corresponding boundary condition as well as the operational condition for the case studied, here is shown in Fig. 3 and Table 2, respectively.

Numerical method: The generation of the computational grid is performed using ANSYS. In order to discretize the three-dimensional unsteady incompressible Navier-Stokes equation, the finite volume methos is applied. A threedimensional pressure-based solver in a first order implicit steady formulation is adoped. Simple C algorithm is used to solve the pressure and velocity coupling. For the convection terms for momentum, turbulent kinetic energy and turbulent energy dissipation rate equations, a second order upwind discretiztion scheme is used.
Table 1: The size of computational domain in D (D = Diameter of the artificial reef unit)

\begin{tabular}{ll}
\hline Variables & Size \\
\hline Length of domain $\left(\mathrm{P}_{\mathrm{p}}\right)$ & $115 \mathrm{D}$ \\
Distance between the reference line and shore line $\left(\mathrm{S}_{\mathrm{c}}\right)$ & $100 \mathrm{D}$ \\
Depth of domain in inlet section $\left(\mathrm{T}_{\mathrm{p}}\right)$ & $5 \mathrm{D}$ \\
Height of AR unit $\left(\mathrm{T}_{\mathrm{c}}\right)$ & $3 \mathrm{D}$ \\
Angle of the bottom, $(\theta)$ & $2.7^{0}$ \\
\hline
\end{tabular}

Table 2: Parameter data used in this simulation

\begin{tabular}{ll}
\hline Physical parameters & Values \\
\hline Velocity of sea water (at inlet, & $1(\mathrm{~m} / \mathrm{sec})$ \\
left, right and top section) & \\
Operating pressure & $101.325(\mathrm{~Pa})$ \\
Density of sea water & $1.025\left(\mathrm{~kg} / \mathrm{m}^{3}\right)$ \\
Viscosity of sea water & $0.001003(\mathrm{~kg} / \mathrm{msec})$ \\
\hline
\end{tabular}


Fig. 1: Schematic of the computational domain containing 3 rows of artificial reefs with parallel formation in horizontal and vertical view (Note: $\mathrm{P}_{p}$ is length of domain, $\mathrm{S}_{\mathrm{c}}$ is distance between the reference line and shore line, $T_{p}$ is depth of domain in inlet section, $T_{c}$ is height of $A R$ unit, $R_{c}$ is the spacing between $\mathrm{AR}$ units in longitudinal direction and $\theta$ is angle of the bottom)



Fig. 2: Artificial reef with 3 rows with parallel formation in horizontal view



Fig. 3: Boundary conditions of CFD Model in isometric view 


\section{RESULTS AND DISCUSSION}

In the present study, the flow fields represented by velocity profile for various artificial reef arangements are analyzed by CFD analysis. The effect of the streamwise and parallel spacing between AR units on the flow fields are explored in detail. The artificial reef arangements considered here is the tube artificial reef with parallel formation varying number of rows and spacing between AR units as discussed as follows.

Effect of spacing: In order to obtain the behavior of sea water flow within and surrounding the artificial reefs, the first investigation is performed dealing with the absence of the artificial reef. Figure 4 shows the velocity profile along the computational domain of the beach area without artificial reef. It is clear that there is no flow behavior identified in this contour. As mentioned in Table 2, the inlet velocity is $1 \mathrm{~m} / \mathrm{sec}$ and therefore, based on Fig. 4, it can be seen that most of local velocities shown in the computational domain refers to the velocity values which are the same with the inlet velocity. As a note, when no barrier of flow current is found, the flow back velocity from the shore line will carry the sand and thus, the abrasion occurs. Therefore, it is believed that to reduce the abrasion, the back velocity must be minimized by introducing and deploying the artificial reefs in best arrangement and in right position relatively to the shore line. As is known in nature the existence of the reef can alter the flow characteristic compared to the condition without the reef. Therefore, it is necessary to deploy the artificial reef, so as it can mimic the reef characteristic.

The main goal of this research is to investigate the optimal arrangement of artificial reef varying spacing between $\mathrm{AR}$ units and also to determine the right position of the deployment of such artificial reef relatively to the shore line. Thus, the optimal length of the slow flow region is of particular interest. Table 3-5 show the comparison of the velocity profile within and surrounding reefs for various arrangements of artificial reef both in vertical and horizontal view. As is shown in Fig. 2, the arrangement of $A R$ is varied by varying spacing between AR units both in longitudinal $\left(\mathrm{R}_{c}\right)$ and transversal $\left(\mathrm{A}_{c}\right)$ direction.

Based on Table 3, there are some specific features dealing with the length of slow flow region $\mathrm{L}_{\mathrm{SF}}$ for the case of Artificial Reef (AR) formation with 1 row. Firstly, in certain condition (in this case when the $\mathrm{A}_{\mathrm{c}}$ is decreased by two times, i.e., from $\mathrm{A}_{c}=1 \mathrm{D}-\mathrm{A}_{\mathrm{c}}=0.5 \mathrm{D}$ ), it seems that
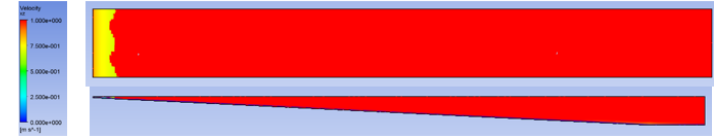

Fig. 4: Velocity profile of the domain without the artificial reef



Fig. 5: Velocity vector in horizontal view for the case of optimal AR arrangement with 3 rows

Table 3: Contour of velocity profile for case of AR arrangement with 1 row

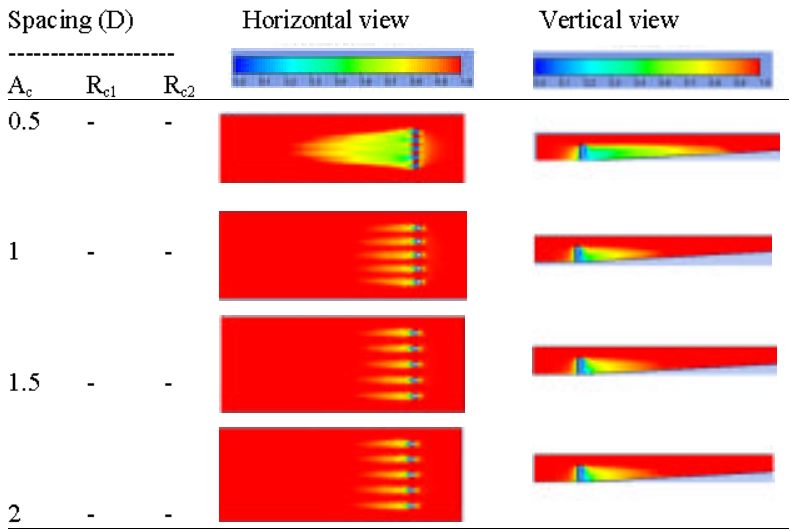

increasing the spacing between $A R$ units $\left(A_{c}\right.$ in this case) decreases the length of the slow flow region. The decrease in the length of slow flow region is reduced up to $33 \%$ when the $\mathrm{A}_{\mathrm{c}}$ is reduced up to $100 \%$. However, for another specific feature, it is found that when the $A_{c}$ is increased from 1-1.5 D, there is no decrease in the length of the slow flow region very much. The difference between the flow characteristic between the case of $\mathrm{A}_{\mathrm{c}}=$ $1 \mathrm{D}$ and the case of $\mathrm{A}_{\mathrm{c}}=1.5 \mathrm{D}$ lies in the width of the slow flow region. It can be observed that the width of the slow flow region becomes smaller when the $\mathrm{A}_{\mathrm{c}}$ is increased. However, for the case of $A_{c}=1.5$ and the case of $A_{c}=2$, 




Table 5: Contour of velocity profile for case of AR arrangement with 3 rows

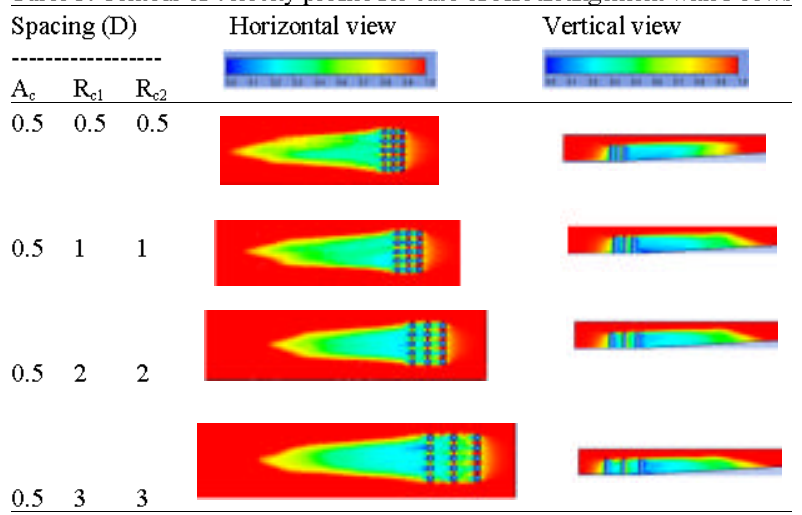

the velocity contour is very similar dealing with the length of the slow flow region as well as the width of the slow flow region. Secondly, increasing the $A_{c}$ to $1 \mathrm{D}$ makes the slow flow region unique for each $\mathrm{AR}$ unit in each row. Based on the ecology point of view, this results in smaller fish zone compared to the $\mathrm{AR}$ with $\mathrm{A}_{\mathrm{c}}=1 \mathrm{D}$. Based on the hydrodynamic point of view, it seems that for preventing the beach abrasion in the case of artificial reef with 1 row, it is suggested to apply the spacing between AR units, $A_{c}$ of $0.5 \mathrm{D}$ due to the longest slow flow region. It is also suggested to deploy such artificial reef formation, so as the distance between the ARs and the shore line is $3 \mathrm{~m}$ as is shown in detail in Table 3 . It should be noted that increased length of slow flow region will attract more fishes and thus enhanced bio-ecosystems.

In the case of $\mathrm{AR}$ with 1 row in real application dealing with the main function of artificial reef, it is likely impossible to deploy the AR with the distance of $3 \mathrm{~m}$ from the shore line. This is because the fact that bio-ecosystem as well as fish cannot live under circumstance in which the flow behavior is not suitable for them. The water temperature near the shore line is also known hotter so much than the other area. Therefore, it is necessary to add more row on the artificial reef with respect to the length of slow flow region.
Table 4 depicts the contour of velocity profile for the case of artificial reef with 2 rows in parallel formation. The computations are performed by varying the $R_{c}$ value (in this case, $0.5-2 \mathrm{D}$ with increment of 0.5 ). It should be noted that for following computations in the case of $\mathrm{AR}$ with 2 rows, it is set that the spacing between AR units in longitudinal direction $\left(A_{c}\right)$ is $0.5 \mathrm{D}$ based on the numerical result as shown in Table 3. From Table 4, it can be observed that the length of the slow flow region $L_{\mathrm{SF}}$ is strongly affected by the spacing between AR units. The longest $\mathrm{L}_{\mathrm{SF}}$ of $6 \mathrm{~m}$ is obtained when the $\mathrm{R}_{\mathrm{c}}$ is $0.5 \mathrm{D}$. When $R_{c}$ is increased by factor 2 , that is from $R_{c}=0.5-R_{c}=1 D$, the $\mathrm{L}_{\mathrm{SF}}$ reduces by $33 \%$. However, further increasing the $\mathrm{R}_{\mathrm{c}}$ does not affect the length of slow flow region. It seems that the trend of flow characteristic in the case of AR with 2 rows is similar to the case of AR with 1 row. There is an optimal spacing in which when this value is increased, the wanted parameter, i.e., slow flow region is not affected by the change of spacing. In comparison to the case of AR with 1 row an interesting thing to note is that for AR with 2 rows, the length of slow flow region is larger (up to $100 \%$ ). It indicates that the $\mathrm{AR}$ with 2 rows is more promising to apply compared to that with 1 row.

With the same frame of the case of artificial reef with 2 rows, Table 5 reflects the comparison of slow flow regions for several arrangements with different $R_{c}$ values for the case of AR with 3 rows. In the present study, it is assumed that the same spacing is applied on the 2 nd and 3rd row of AR units. On the other word, $\mathrm{Rc}_{1}$ value will be equal to $\mathrm{Rc}_{2}$ value (Fig. 2). Unlike the case of AR with 2 rows, increasing the $R_{c}$ does not affect the contour of velocity profile as well as the length of the slow flow region very much. The generated slow flow refers one bigger zone that connects each AR unit. From the ecological and hydrodynamic terms, this generates a best condition for fish and bio-ecosystem. Regarding with the length of the slow flow region, the AR formation with different $R_{c}$ values predicts the same value, that is $6 \mathrm{~m}$. For beach abrasion prevention, this is the wanted condition. The long $\mathrm{L}_{\mathrm{SF}}$ makes the deployment of AR possible to apply in real. The back velocity from the shore will reduce if the AR is deployed in right location, so as the sea water does not carry the sand to the middle of the sea.

Based on Table 3-5, it can be concluded that the arrangement and spacing between artificial reef units strongly affect the velocity distribution (i.e., slow flow region) within and surrounding artificial reef. In detail, Table 6 shows the prediction of the length of slow flow region as well as the minimum velocity within the slow flow region for all AR formations. It is found that the artificial reef with 3 rows gives the best flow behavior with respect to the slow flow region, especially, when the formation used is AR with $\mathrm{Ac}=0.5 \mathrm{R}_{\mathrm{c} 1}=0.5$ and $\mathrm{R}_{\mathrm{c} 2}=$ $0.5 \mathrm{D}$. This formation produces the lowest minimum Table 
Table 6: Flow characteristics for various artificial reef arrangements and spacings

\begin{tabular}{|c|c|c|c|c|}
\hline \multicolumn{5}{|c|}{ AR formation/Distance (D) } \\
\hline$\underline{A c}$ & $\mathrm{R}_{\mathrm{cl}}$ & $\mathrm{R}_{62}$ & $(\mathrm{~m} / \mathrm{sec})$ & Flow region $\mathrm{L}_{\mathrm{SF}}(\mathrm{m})$ \\
\hline \multicolumn{5}{|c|}{ AR with 1 row } \\
\hline 0.5 & - & - & 0.539 & 3 \\
\hline 1 & - & - & 0.680 & 2 \\
\hline 1.5 & - & - & 0.693 & 1 \\
\hline 2 & - & - & 0.732 & 1 \\
\hline \multicolumn{5}{|c|}{ AR with 2 rows } \\
\hline 0.5 & 0.5 & - & 0.473 & 6 \\
\hline 0.5 & 1 & - & 0.447 & 6 \\
\hline 0.5 & 1.5 & - & 0.420 & 6 \\
\hline 0.5 & 2 & - & 0.432 & 6 \\
\hline 0.5 & 2,5 & - & 0.582 & 6 \\
\hline 1 & 0.5 & - & 0.750 & 4 \\
\hline 1 & 1 & - & 0.676 & 4 \\
\hline 1 & 1.5 & - & 0.639 & 4 \\
\hline 1 & 2 & - & 0.632 & 4 \\
\hline 1.5 & 0.5 & - & 0.842 & 4 \\
\hline 1.5 & 1 & - & 0.780 & 4 \\
\hline 1.5 & 1.5 & - & 0.782 & 4 \\
\hline 1.5 & 2 & - & 0.753 & 4 \\
\hline 2 & 0.5 & - & 0.862 & 4 \\
\hline 2 & 1 & - & 0.858 & 4 \\
\hline 2 & 1.5 & - & 0.799 & 4 \\
\hline 2 & 2 & - & 0.814 & 4 \\
\hline \multicolumn{5}{|c|}{ AR with 3 rows } \\
\hline 0.5 & 0.5 & 0.5 & 0.458 & 10 \\
\hline 0.5 & 1 & 1 & 0.413 & 10 \\
\hline 0.5 & 1,5 & 1,5 & 0.323 & 10 \\
\hline 0.5 & 2 & 2 & 0.450 & 10 \\
\hline 0.5 & 2,5 & 2,5 & 0.644 & 10 \\
\hline
\end{tabular}



Fig. 6: Velocity vector in vertical view for the case of optimal AR arrangement with 3 rows

velocity of $0.235 \mathrm{~m} / \mathrm{sec}$ which means that such formation can reduce the velocity up to $76.5 \%$ at the farthest distance of $10 \mathrm{~m}$. In his way, the beach abrasion can be reduced significantly.

Velocity vector: Figure 5 shows the velocity vector surrounding reefs with 3 rows in parallel formation which is in this case an optimal AR formation dealing with the length of slow flow region. It can be observed that in around tube reefs, the flow has lower velocities than other region, especially, the flow fields behind the artificial reef. This is expected as the hydrodynamics resulted in surrounding reef produces the back vortex which decelerates the water velocities hitting the reef as seen in detail in Fig. 6. On the other words, this physical phenomenon leads to the stability of artificial reef structure and it makes the deployment of the artificial reefs more reasonable.

\section{CONCLUSION}

In the present study, the three-dimensional turbulent flow within and surrounding tube artificial reef was simulated based on computational fluid dynamic. Different arrangements of artificial reefs varying spacing between $\mathrm{AR}$ units both in longitudinal and transversal direction were explored with respect to the length of slow flow region. Some main conclusions are as follows: The closer the spacing between artificial reef units, the longer the length of slow flow region. Increasing the number of row in artificial reef formation enhances the length of slow flow region

The optimal formation of artificial reef with respect to the length of slow flow region is the formation with 3 rows in which $A_{c}=0.5, R_{c 1}=0.5$ and $R_{c 2}=0.5 \mathrm{D}$. It is also suggested to deploy such AR formation at a distance of $10 \mathrm{~m}$ behind the last row of $\mathrm{AR}$ to reduce the beach abrasion

\section{ACKNOWLEDGEMENTS}

This research is fully supported by RPI (Research Publication International) grant, No. 831.1-01/UN7.P4.3/ $\mathrm{PP} / 2017$. The researchers fully acknowledged Institute for Research and Community Services (LPPM) Diponegoro University for the approved fund which makes this important research viable and effective.

\section{REFERENCES}

Bohnsack, J.A. and D.L. Sutherland, 1985. Artificial reef research: A review with recommendations for future priorities. Bull. Marine Sci., 37: 11-39.

Jiang, Z., Z. Liang, Y. Liu, Y. Tang and L. Huang, 2013. Particle image velocimetry and numerical simulations of the hydrodynamic characteristics of an artificial reef. Chin. J. Oceanol. Limnol., 31: 949-956.

Jiang, Z., Z. Liang, Y. Tang, L. Huang and D. $\mathrm{Yu}$ et al., 2010. Numerical simulation and experimental study of the hydrodynamics of a modeled reef located within a current. Chin. J. Oceanol. Limnol., 28: 267-273.

Jiao, L., Z. Yan-Xuan, G. Pi-Hai and G. Chang-Tao, 2017. Numerical simulation and PIV experimental study of the effect of flow fields around tube artificial reefs. Ocean Eng., 134: 96-104. 
Kim, D., J. Woo, H.S. Yoon and W.B. Na, 2014. Wake lengths and structural responses of Korean general artificial reefs. Ocean Eng., 92: 83-91.

Liu, T.L. and D.T. Su, 2013. Numerical analysis of the influence of reef arrangements on artificial reef flow fields. Ocean Eng., 74: 81-89.

Liu, Y., C.T. Guan, Y.P. Zhao, Y. Cui and G.H. Dong, 2012. Numerical simulation and PIV study of unsteady flow around hollow cube artificial reef with free water surface. Eng. Appl. Comput. Fluid Mech., 6: 527-540.

Liu, Y., Y.P. Zhao, G.H. Dong, C.T. Guan and Y. Cui et al., 2013. A study of the flow field characteristics around star-shaped artificial reefs. J. Fluids Struct., 39: 27-40.

Miao, Z.Q. and Y.H. Xie, 2007. Effects of water-depth on hydrodynamic force of artificial reef. J. Hydrodyn. Ser. B, 19: 372-377.
Ontowirjo, B. and H.D. Armono, 2003. Artificial Reef Methodology for Coastal Protection using Submerged Structures: A Numerical Modeling Approach. In: Soft Shore Protection, Goudas, C., G. Katsiaris, V. May and T. Karambas (Eds.). Springer, Dordrecht, TheNetherlands,ISBN:978-94-01 0-3966-6, pp: 211-225.

Suzuki, G., S. Kai, H. Yamashita, K. Suzuki and Y. Iehisa et al., 2011. Narrower grid structure of artificial reef enhances initial survival of in situ settled coral. Marine Pollut. Bull., 62: 2803-2812.

Woo, J., D. Kim, H.S. Yoon and W.B. Na, 2014. Characterizing Korean general artificial reefs by drag coefficients. Ocean Eng., 82: 105-114. 